\title{
Bayesian decay time estimation in a reverberation chamber for absorption measurements
}

\author{
Balint, Jamilla; Muralter, Florian; Nolan, Melanie; Jeong, Cheol-Ho
}

Published in:

Journal of the Acoustical Society of America

Link to article, DOI:

$10.1121 / 1.5125132$

Publication date:

2019

Document Version

Publisher's PDF, also known as Version of record

Link back to DTU Orbit

Citation (APA):

Balint, J., Muralter, F., Nolan, M., \& Jeong, C-H. (2019). Bayesian decay time estimation in a reverberation chamber for absorption measurements. Journal of the Acoustical Society of America, 146(3), 1641-1649. https://doi.org/10.1121/1.5125132

\section{General rights}

Copyright and moral rights for the publications made accessible in the public portal are retained by the authors and/or other copyright owners and it is a condition of accessing publications that users recognise and abide by the legal requirements associated with these rights.

- Users may download and print one copy of any publication from the public portal for the purpose of private study or research.

- You may not further distribute the material or use it for any profit-making activity or commercial gain

- You may freely distribute the URL identifying the publication in the public portal

If you believe that this document breaches copyright please contact us providing details, and we will remove access to the work immediately and investigate your claim. 


\section{Bayesian decay time estimation in a reverberation chamber for absorption measurements}

Jamilla Balint, Florian Muralter, Mélanie Nolan, and Cheol-Ho Jeong

Citation: The Journal of the Acoustical Society of America 146, 1641 (2019); doi: 10.1121/1.5125132

View online: https://doi.org/10.1121/1.5125132

View Table of Contents: https://asa.scitation.org/toc/jas/146/3

Published by the Acoustical Society of America

\section{ARTICLES YOU MAY BE INTERESTED IN}

Talker change detection: A comparison of human and machine performance

The Journal of the Acoustical Society of America 145, 131 (2019); https://doi.org/10.1121/1.5084044

Individual differences in the acoustic properties of human skulls

The Journal of the Acoustical Society of America 146, EL191 (2019); https://doi.org/10.1121/1.5124321

The relative size of auditory scenes of multiple talkers

The Journal of the Acoustical Society of America 146, EL219 (2019); https://doi.org/10.1121/1.5125007

Echolocation signals of free-ranging pantropical spotted dolphins (Stenella attenuata) in the South China Sea The Journal of the Acoustical Society of America 145, 3480 (2019); https://doi.org/10.1121/1.5111742

Non-symmetric flexural wave scattering and one-way extreme absorption

The Journal of the Acoustical Society of America 146, 873 (2019); https://doi.org/10.1121/1.5087133

Pseudospins and topological edge states for fundamental antisymmetric Lamb modes in snowflakelike phononic crystal slabs

The Journal of the Acoustical Society of America 146, 729 (2019); https://doi.org/10.1121/1.5114903 


\title{
Bayesian decay time estimation in a reverberation chamber for absorption measurements
}

\author{
Jamilla Balint ${ }^{a)}$ \\ Signal Processing and Speech Communication Laboratory, Department of Electrical Engineering, \\ Graz University of Technology, Inffeldgasse. 16c, A-8010 Graz, Austria \\ Florian Muralter \\ Computing, Electronics and Communication Technologies, Faculty of Engineering, University of Deusto, \\ Bilbao, Spain \\ Mélanie Nolan ${ }^{\text {b) }}$ and Cheol-Ho Jeong \\ Acoustic Technology, Department of Electrical Engineering, Technical University of Denmark (DTU), \\ Building 352, Ørsteds Plads, DK-2800 Kongens Lyngby, Denmark
}

(Received 20 April 2019; revised 13 August 2019; accepted 19 August 2019; published online 19 September 2019)

This work investigates the use of the initial decay time to obtain the Sabine absorption coefficient from measurements conducted in a reverberation chamber. Due to non-uniform distribution of sound absorption in the test chamber, measured energy decay functions exhibit multiple slopes, which cannot be evaluated unambiguously using linear regression as prescribed in the current standard (ISO 354, International Organization for Standardization, Geneva, Switzerland, 2003). As an alternative, this study proposes a Bayesian framework that allows estimating multiple decay parameters, hence capturing more accurately the energy decay features. Measurements are carried out in a reverberation chamber with and without diffusing elements to investigate the influence of diffusers on the absorption coefficient and on the decay process. Measured absorption coefficients of a porous sample are compared to theoretical values estimated with a transfer matrix model. The results show that the Sabine absorption coefficient calculated using the shortest decay time agrees well with the size-corrected theoretical absorption coefficient.

() 2019 Acoustical Society of America. https://doi.org/10.1121/1.5125132

Pages: $1641-1649$

\section{INTRODUCTION}

The Sabine absorption coefficient can be obtained from measurements in a reverberation chamber. ${ }^{1}$ The procedure of calculating single reverberation times (RTs) is based on Sabine's formula, ${ }^{2}$ which assumes a diffuse sound field and an exponential energy decay. Yet, when absorption is concentrated on a single surface, the energy decay does not decay exponentially but exhibits a double slope (in the logarithmic presentation), of which the decay rates correspond to those of the grazing and non-grazing waves. ${ }^{3}$ This means that different RTs will be obtained depending on what portion of the decay function is used for the evaluation, and therefore the decay process cannot be described by a single RT ${ }^{4,5}$ Consequently, absorption coefficients obtained under the conditions specified in the standard might be ambiguous. This poses a major challenge, since results obtained from measurements in the reverberation chamber should be reproducible as they form the basic input parameter for room acoustic simulation tools and acoustic design processes. Yet, numerous investigations have reported significant doubts regarding the reliability and accuracy of the reverberation chamber method. In particular, several round robin tests, in which the same sample of an absorbing material has been tested in a number of

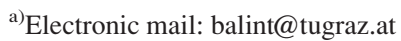

b) Also at: Saint-Gobain Ecophon, 26575 Hyllinge, Sweden.
}

laboratories, have revealed a substantial discrepancy in the results. ${ }^{6-8}$ This could, to some extent, be attributed to the estimation of the RT from multi-exponential decay functions with the linear regression (LR) method.

The measurement standard prescribes specific design criteria for reverberation chambers. ${ }^{1}$ Especially, adding diffusing elements (in the form of panel or boundary diffusers) is expected to build up diffusion, and thus to minimize the curvature of the sound decays. However, predicting the effect of diffusing elements on the sound field in a reverberation chamber is a challenging task. Frameworks like the finite element method, the boundary element method, or geometrical acoustics simulation tools depend critically on the boundary conditions, which are often unknown. ${ }^{10}$ Furthermore, geometrical acoustics simulation tools cannot take into account wavebased effects. $^{9}$

Uncertainties in standardized reverberation chamber measurements are not only introduced by uneven distribution of absorption. ISO 354 (Ref. 1) further stipulates that the evaluation range shall start at $5 \mathrm{~dB}$ below the initial sound pressure level. Kuttruff ${ }^{4}$ concluded that in the case of a multi-exponential decay, the initial part of the decay contains the mean of all excited modes in the room, and therefore the most relevant information. He derived that the decay function can be seen as the Laplace transform of the damping distribution of the room modes. By calculating the first and second derivatives of the decay distribution, he 
concluded that the initial slope of a logarithmically plotted decay function is defined by the centroid of the distribution. Therefore the initial slope is the weighted mean of all decay constants present in the reverberation process, where the weighting function is the frequency distribution of the decay constants. However, the current evaluation procedure ${ }^{1}$ discards the initial portion of the sound decay and therefore the accurate estimation of reverberation parameters in case of multi-exponential decays may not be possible.

The major contribution of this work is to calculate the Sabine absorption coefficient with accurate decay parameters obtained from multi-exponential energy decay functions (EDFs) measured in a reverberation chamber. In case of multiexponential decays, a Bayesian framework is used for estimating accurate decay parameters within this investigation. The absorption coefficient is calculated with the decay time obtained from the initial part of the EDF. Results are compared to theoretical absorption coefficients calculated with a transfer matrix model $^{11}$ combined with Miki's model ${ }^{12}$ and Thomasson's size correction. ${ }^{13}$

This paper is organized as follows: Sec. II gives an overview of previous research and underlying ideas of the present work. The Bayesian framework to obtain multiple decay parameters is presented in Sec. III. Experimental results are described in Sec. IV and discussed in Sec. V. Finally, Sec. VI summarizes the work and gives an outlook for future research on the presented topic.

\section{PREVIOUS RESEARCH}

EDFs in enclosed spaces of various sizes and shapes have widely been investigated in the past. Results indicate that in numerous cases a multi-exponential decay is present. Hunt et al. ${ }^{3}$ showed that the root-mean-square sound pressure at a given point during the decay is the summation of each excited normal mode of vibration. In reverberation chambers with uneven distribution of absorption, the large number of excited modes within a given frequency band has to be subdivided into groups having common properties, leading to multiple decay parameters. Although restricted to rectangular rooms without any diffusing objects, the proposed method could be validated by various experiments. It was suggested not to use any diffusing elements in the reverberation chamber since the effect on the sound field cannot be predicted and poses the risk of introducing additional uncertainties. When introducing the backward integration method, Schroeder also pointed out the existence of multi-exponential decays. ${ }^{14}$ As an example, he published measurements carried out in the Boston Symphony Hall exhibiting multiple decay times. Bruel ${ }^{15}$ observed concave sound decays when trying to determine RTs for sound power measurements in diffuse-field conditions. He found out that the agreement between the freeand diffuse-field sound powers is much better when the early decay is used. Jacobsen ${ }^{16}$ derived decay times of each mode group and showed that axial and tangential modes exhibit a longer decay time than oblique modes. In small and mediumsized rooms, the decay will therefore always be multiplesloped at low frequencies. Furthermore he pointed out that if the initial part of the decay is used for calculating the absorption coefficient, care has to be taken at low frequencies where the sound field is dominated by axial and tangential modes, as they contribute significantly to the steady-state condition in the room. ${ }^{17}$ Still, he concluded that the systematic error of absorption estimates based on initial decay rates is about two-thirds of the error of usual estimates based on the interval from -5 to $-35 \mathrm{~dB}$. In ISO $3382-2,{ }^{18}$ a parameter for the linearity of the decay is proposed, which estimates the curvature based on the ratio of the RTs $T_{20}$ and $T_{30}$. It has to be pointed out that this gives a rough estimation of whether or not a multi-exponential decay is present, although neglecting the bending point of the curve. Nilsson ${ }^{5}$ investigated rectangular rooms with absorbing ceilings like classrooms and offices. He successfully applied a two-system statistical energy analysis model to simulate the double-sloped energy decay due to the uneven distribution of absorption. Furthermore the influence of diffusing objects was examined, showing that the curvature can be decreased and the bending point of the decay shifted when introducing irregularities at the boundaries such as boxes on the walls. ${ }^{19}$ Preliminary studies ${ }^{20,21}$ to the present manuscript were carried out, where the influence of different amounts of diffusing objects on the bending point of the EDF has been investigated in reverberation chambers. Xiang et al. ${ }^{22-25}$ thoroughly investigated the decay behaviour in coupled spaces and developed a Bayesian framework to calculate multiple decay parameters. The successful application resulted in up to tripleslope detections, showing that the use of conventional methods of LR is not valid in those cases.

Previous research, as shown, indicates that multiexponential decays are ubiquitous: in single and coupled spaces, in concert halls, in small rooms, in rooms with uneven distribution of absorption, in classrooms, and in laboratories. Although it was pointed out that in cases of multi-exponential decays the determination of RTs with LR can lead to questionable results, ${ }^{22-25}$ the standard procedure for calculating absorption coefficients still neglects the curved nature of energy decays which is apparent in the reverberation chamber.

\section{FRAMEWORK}

This section describes the Bayesian framework used to calculate the decay time distribution of a given EDF. Bayesian frameworks were already successfully applied to estimate multiple decay parameters by Xiang et al. ${ }^{22-25}$ in the field of acoustics and by Shukla et al..$^{26-28}$ and Hoffmann et $a .^{29}$ in the field of physics.

\section{A. Bayesian decay time estimation}

Bayes' theorem provides a probabilistic framework to solve inverse problems in a variety of fields including acoustics, ${ }^{25,30,31}$ image processing, ${ }^{32}$ and physics. ${ }^{26-28}$ In this work, the aim is to calculate the decay time distribution from a given EDF. The method of extracting decay times is based on the algorithm MELT_Maximum Entropy LifeTime Analysis, ${ }^{26-29}$ which was used in the field of positron lifetime analysis. The authors employed the framework to solve the inverse problem of finding the decay times and their intensities resulting from measured multi-exponential decay functions. In acoustics, the EDF, which is obtained by applying the Schroeder backward integration to 
an impulse response (IR), can be regarded as a multiexponential decay function as well, ${ }^{25}$

$$
\operatorname{EDF}\left(A, T, t_{k}\right)=A_{0}\left(t_{K}-t_{k}\right)+\sum_{i=1}^{N} A_{i} \cdot e^{-13.8 \cdot t_{k} / T_{i}},
$$

where the linear term $A_{0}\left(t_{K}-t_{k}\right)$ is associated with the noise in the room IR, $t_{k}$ is the time parameter, $t_{K}$ is the total length of the IR, and $k=1,2, \ldots, K . A_{i}$ denotes the linear amplitude of the $i$ th exponential decay function, $T_{i}$ is the decay time of the $i$ th decay function, and $N$ corresponds to the total number of slopes. The algorithm MELT is modified to directly extract decay times $T_{i}$ and corresponding intensities $A_{i}$ from an EDF. Detailed mathematical formulations are presented in the Appendix, following the derivations of Shukla et al. ${ }^{26-28}$ and Hoffmann et al. ${ }^{29}$

\section{B. Numerical example}

To illustrate how decay times from multi-exponential data can be obtained, an artificial EDF is constructed. The decay process is modelled according to Eq. (1) and consists

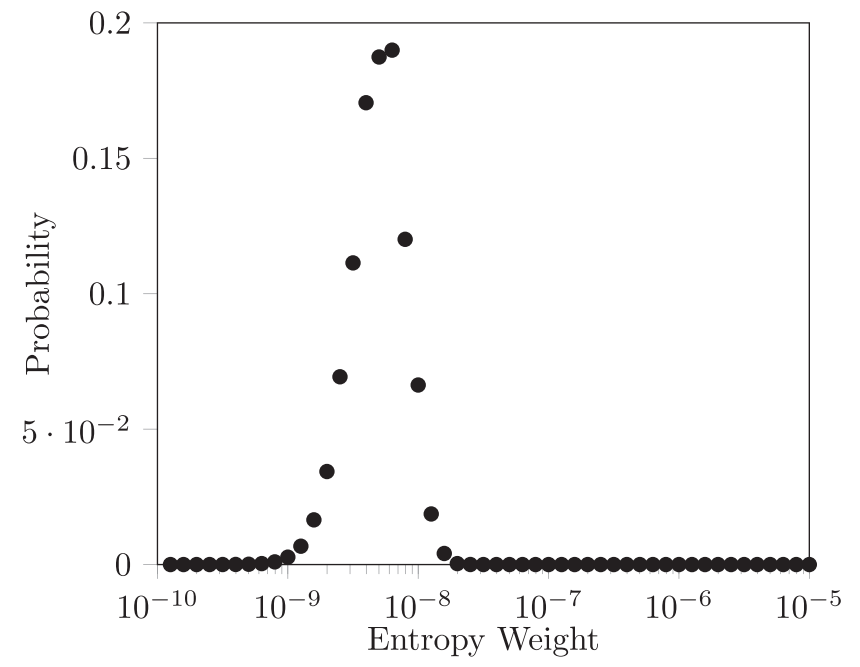

(A) of 100 exponentially decaying functions and an additive noise term. The decay times are normally distributed with mean values of $T_{1}=1.40 \mathrm{~s}$ and $T_{2}=4.60 \mathrm{~s}$ and a standard deviation of 0.1 , resulting in a double-slope behaviour. The amplitude of the noise is set to $A_{0}=5 \times 10^{-9}$, and the amplitudes of the decays are set to $A_{1}=0.70$ and $A_{2}=0.30$. This data are fed to the algorithm to estimate decay times from the given EDF. Through variation of the entropy weights the result with the highest probability is obtained [see Fig. 1(A)]. The result of the estimated decay times with the highest probability is shown in Fig. 1(B), where the normalized intensity spectrum (which corresponds to the linear amplitude of the decay process) is represented as a function of decay time. The local maxima of the decay times in Fig. 1(B) correspond to the mean values of the normal distributions used to obtain the artificial EDF. The peak values of the local maxima (decay times $T_{i}$ and amplitudes $A_{i}$ ) are summed up to model the artificial EDF. The artificial EDF as well as the EDF obtained with the estimated decay times are shown in Fig. 1(C). The decay process is modelled with two exponential functions with the

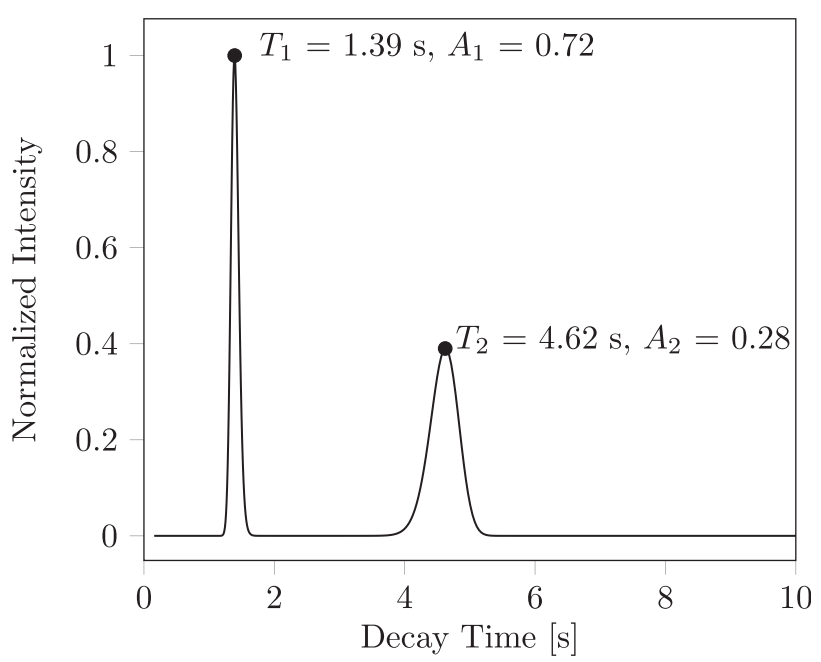

(B)

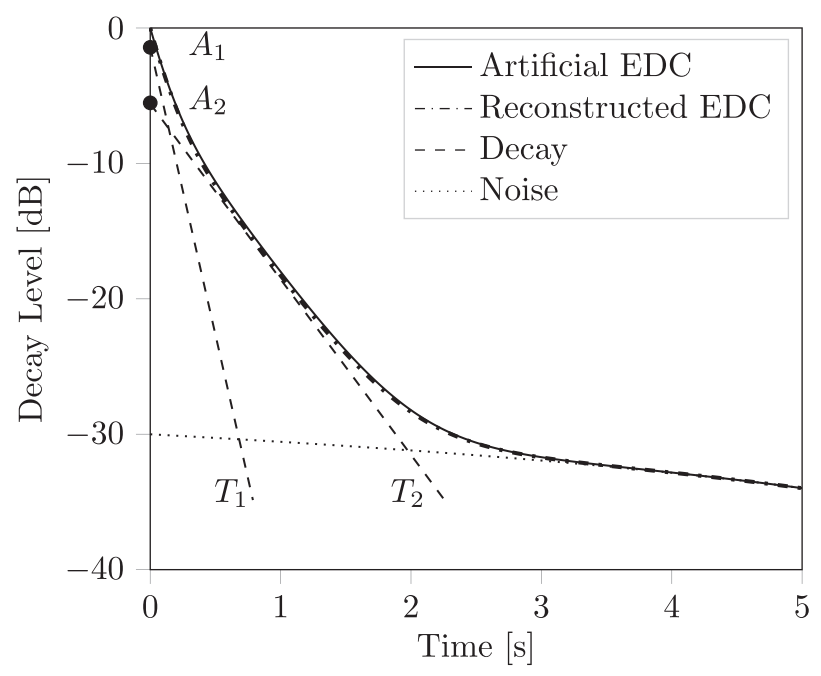

(C)

FIG. 1. (A) Variation of the entropy weights, (B) intensity spectrum of the estimated decay times, (C) artificial and reconstructed energy decay curve with the peaks of the intensities $A_{1}=0.72, A_{2}=0.28, T_{1}=1.39 \mathrm{~s}, T_{2}=4.62 \mathrm{~s}$, and an additive noise term at $A_{0}=5 \times 10^{-9}$. 
amplitudes $A_{1}=0.72, A_{2}=0.28$, the corresponding decay times $T_{1}=1.39 \mathrm{~s}$ and $T_{2}=4.62 \mathrm{~s}$, and an additive noise term. In practice, if absorption is concentrated on a single surface, the room modes can be separated into two groups ${ }^{5,16}$ (more attenuated modes and less attenuated modes). The first peak, which corresponds to the shortest decay time, can be regarded as the modes which are attenuated the most. Therefore the shortest decay time is used when calculating the absorption coefficient in Sec. IV.

\section{EXPERIMENTAL RESULTS}

This section describes experimental measurements conducted to validate the benefit of using the proposed Bayesian framework for decay time estimation and absorption coefficient measurements. Absorption coefficients obtained with conventional reverberation parameters will be compared to values obtained with the Bayesian framework. Additionally, a theoretical absorption coefficient serves as a reference value for comparison.

\section{A. Measurements}

Measurements are carried out in a box-shaped reverberation chamber $\left(V=241 \mathrm{~m}^{3}\right)$ at the Technical University of Denmark. The absorption coefficient of a porous sample is measured according to ISO 354 (Ref. 1) in the chamber with 20 panel diffusers and without diffusers. The porous sample with a flow resistivity of $12.9 \mathrm{kPa} \mathrm{s} / \mathrm{m}^{2}$ has a total area of $10.8 \mathrm{~m}^{2}(3.6 \mathrm{~m} \times 3.0 \mathrm{~m} \times 0.1 \mathrm{~m})$ and its sides are covered by a wooden frame. Figure 2 shows the room setup, with the porous absorber placed on the floor of the chamber and acrylic panel diffusers hung from the ceiling. IRs are measured at 12 independent source-receiver positions and the EDF is calculated by Schroeder backward integration for each IR. ${ }^{14}$ EDFs are averaged over all measurement positions to obtain a single function. Conventional reverberation parameters like the early decay time (EDT), $T_{20}$, and $T_{30}$ are calculated by applying LR to decay ranges 0 to $-10 \mathrm{~dB}$,

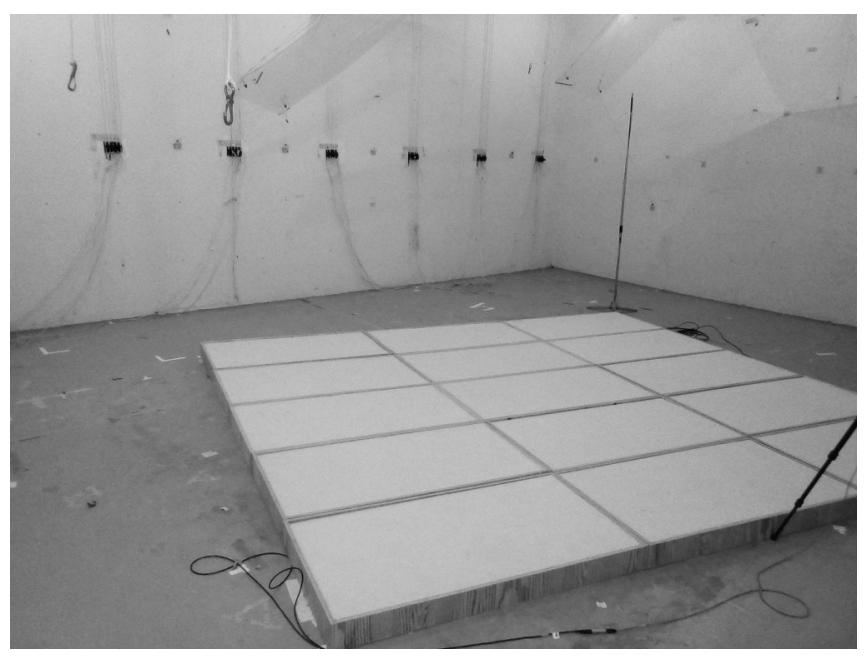

FIG. 2. Porous absorber placed on the floor in the reverberation chamber $\left(V=241 \mathrm{~m}^{3}\right)$, absorber with sides covered by a wooden frame and a total area of $S=10.8 \mathrm{~m}^{2}$.
-5 to $-25 \mathrm{~dB}$, and -5 to $-35 \mathrm{~dB}$, respectively. The initial decay time is obtained with the proposed framework.

The equivalent absorption area $A$ of a porous sample is calculated according to ${ }^{1}$

$$
A=\frac{55.3 \cdot V}{c} \cdot\left(\frac{1}{T_{e}}-\frac{1}{T_{a}}\right)-4 \cdot V \cdot m,
$$

where $V$ denotes the total volume of the chamber, $c$ is the speed of sound, $m$ is the attenuation coefficient, $T_{e}$ is the RT in the undamped chamber (empty), and $T_{a}$ is the RT in the damped chamber (with the absorber). The Sabine absorption coefficient for octave bands from 125 to $4000 \mathrm{~Hz}$ is calculated according to

$$
\alpha=\frac{A}{S},
$$

where $S$ denotes the total area of the sample.

\section{B. Theoretical absorption coefficient}

The random incidence absorption coefficient for an infinite absorber can be calculated as follows: ${ }^{33,34}$

$$
\alpha_{\text {random }}=\int_{0}^{\pi / 2} \alpha_{\text {inf }}\left(\Theta_{i}\right) \sin \left(2 \Theta_{i}\right) d \Theta_{i},
$$

where $\alpha_{\text {inf }}\left(\Theta_{i}\right)$ is the oblique incidence absorption coefficient at incidence angle $\Theta_{i}$.

In a reverberation chamber, the measured absorption coefficient is typically overestimated due to diffraction phenomena evoked by the edges of the finite-size sample. This size-effect is particularly marked at low frequencies (below $1 \mathrm{kHz}$ ). A size-corrected random incidence absorption coefficient is derived by Thomasson ${ }^{13}$ as follows:

$$
\alpha_{\text {theory }}=2 \int_{0}^{\pi / 2} \frac{4 \operatorname{Re}\left(Z_{s}\right)}{\left|Z_{s}+\bar{Z}_{r}\right|^{2}} \sin (\Theta) d \Theta,
$$

where $Z_{s}$ denotes the surface impedance and $\overline{Z_{r}}$ the radiation impedance which is a function of shape, size, frequency, and is averaged over azimuthal angles from 0 to $2 \pi$. The main assumptions are local reaction, infinite baffle, and flushmounting of the absorber to the infinite baffle. Although the theoretical absorption coefficient cannot be regarded as the "true" absorption coefficient of the sample, it is chosen to be a reference in this paper for the following reasons. Such a coefficient is a comprehensible comparison value as opposed to ISO results. Several round robin tests have shown large deviations in the results from chamber to chamber, raising serious doubts regarding the reliability of the obtained coefficients. ${ }^{6-8}$ Besides, the derivation by Thomasson makes it possible to account for the finite size of the sample and has therefore been widely used in the literature. ${ }^{35-38}$

In the following, $\alpha_{\text {theory }}$ in Eq. (5) is calculated based on a transfer matrix model for the case of a single layer of porous material with a rigid backing. ${ }^{11}$ The surface impedance and complex wave number are calculated with Miki's model, ${ }^{12}$ which is a modified version of the Delany and Bazley 
TABLE I. Theoretical size-corrected absorption coefficient for a porous absorber with a flow resistivity of $12.9 \mathrm{kPa} s / \mathrm{m}^{2}$ calculated with Miki's model and Thomasson's size correction.

\begin{tabular}{lllllll}
\hline \hline$f(\mathrm{~Hz})$ & 125 & 250 & 500 & 1000 & 2000 & 4000 \\
\hline$\alpha_{\text {theory }}$ & 0.86 & 1.24 & 1.14 & 1.03 & 0.98 & 0.98 \\
\hline \hline
\end{tabular}

model,${ }^{39}$ and gives improved results at low frequencies. The calculated results for the theoretical absorption coefficients are shown in Table I. Thomasson's size-corrected absorption coefficient for a finite sample of $3.6 \mathrm{~m} \times 3 \mathrm{~m} \times 0.1 \mathrm{~m}$ backed by a rigid wall exceeds unity for the octave bands centered at $250 \mathrm{~Hz}, 500 \mathrm{~Hz}$, and $1 \mathrm{kHz}$.

For comparison, the deviation $\Delta \alpha$ of the measured absorption coefficient from the theoretical absorption coefficient $\alpha_{\text {theory }}$ is calculated with

$$
\Delta \alpha=\alpha_{\text {theory }}-\alpha,
$$

where $\alpha$ is the Sabine absorption coefficient calculated according to Eqs. (2) and (3) with different reverberation parameters: EDT, $T_{20}, T_{30}$, and the decay time $T_{1}$.

\section{Linear regression (LR)}

To illustrate how conventional reverberation parameters EDT, $T_{20}$, and $T_{30}$ vary in case of multi-exponential data, results are shown for one frequency band. In Fig. 3 the energy decay curve, as well as the regression lines for the damped condition at $500 \mathrm{~Hz}$, are shown with and without panel diffusers. The decay times EDT, $T_{20}$, and $T_{30}$ vary from 2.90 to $3.44 \mathrm{~s}$ without diffusing elements, indicating a multi-exponential decay due to the increase of the RT when the evaluation range is increased. When diffusing elements are added to the room, the reverberation parameters EDT, $T_{20}$, and $T_{30}$ vary from 2.12 to $2.21 \mathrm{~s}$, still indicating a slightly curved decay but much less than in the case without

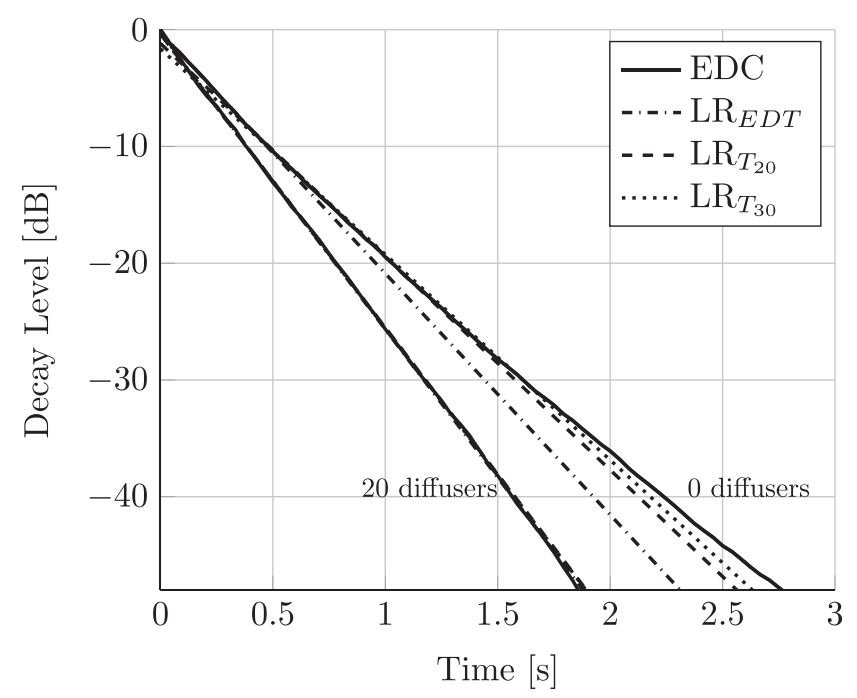

FIG. 3. Energy decay curve and LR for $f=500 \mathrm{~Hz}$ in the damped condition for room acoustic parameter without diffusers EDT $=2.90 \mathrm{~s}, T_{20}=3.29 \mathrm{~s}$, $T_{30}=3.44 \mathrm{~s}$, and with diffusers EDT $=2.12 \mathrm{~s}, T_{20}=2.21 \mathrm{~s}, T_{30}=2.21 \mathrm{~s}$.
TABLE II. Absorption coefficient $\alpha$ for the $500 \mathrm{~Hz}$ band calculated with different RTs: EDT, $T_{20}$, and $T_{30}$, with and without diffusers.

\begin{tabular}{lccc}
\hline \hline RT $/ \alpha$ & EDT & $T_{20}$ & $T_{30}$ \\
\hline$\alpha$ with 0 diffusers & 0.86 & 0.8 & 0.7 \\
$\alpha$ with 20 diffusers & 1.20 & 1.18 & 1.18 \\
\hline \hline
\end{tabular}

diffusers. The RT is decreased significantly compared to the case without panels, since the sound waves are redirected onto the absorber by the panels. $T_{30}$ is decreased from 3.44 to $2.12 \mathrm{~s}$ when diffusers are added, also EDT is reduced from 2.90 to $2.12 \mathrm{~s}$. The results are in agreement with investigations carried out in Ref. 19.

To investigate how the evaluation range for reverberation parameters influences the absorption coefficient, $\alpha$ is calculated for $f=500 \mathrm{~Hz}$ with EDT, $T_{20}$, and $T_{30}$ with and without diffusing elements. Table II shows the absorption coefficients calculated with EDT, $T_{20}$, and $T_{30}$. The values for $\alpha$ vary between 0.70 and 0.86 without diffusing elements and from 1.18 to 1.20 with diffusing elements. The absorption coefficient decreases when the evaluation range for the decay parameters is increased. Therefore the absorption coefficient is higher when EDT is used instead of $T_{20}$ or $T_{30}$. This is in agreement with results in Ref. 4, where the author shows that the initial part of the EDF contains the mean of all excited modes and should be used to calculate $\alpha$ rather than the late part of the EDF. Furthermore the author concludes that the later part of the EDF only contains the less attenuated modes, which will lead to questionable results when used to calculate the absorption coefficient.

Table III shows the absorption coefficients without any diffusing elements, calculated from $125 \mathrm{~Hz}$ to $4 \mathrm{kHz}$ with the reverberation parameter $T_{20}$ (ISO 354 stipulates to use $T_{20}$ ) and the deviation from the theoretical values. The absorption coefficients are lower than the theoretical values in the entire frequency range. Due to the multi-exponential nature of the EDF, using LR to obtain $T_{20}$ will underestimate the absorption coefficient.

Table IV shows the RTs $T_{20}$ and the absorption coefficient obtained with diffusers. In this case the absorption coefficient is increased and exceeds the theoretical values at the $500 \mathrm{~Hz}$ band and above. At the $250 \mathrm{~Hz}$ band the absorption coefficient is significantly increased compared to the case without diffusing elements from 0.75 to 1.03 . At the $125 \mathrm{~Hz}$ band the absorption coefficient is slightly increased from 0.37 to 0.39 . It is expected that the absorption coefficient increases when diffusing elements are added to the chamber because the sound waves are redirected onto the absorber by the

TABLE III. RT $T_{20}$ in the undamped $\left(T_{20, \text { empty }}\right)$ and damped $\left(T_{20, \text { absorber }}\right)$ conditions, resulting absorption coefficient and deviation $\Delta \alpha$. No diffusers are installed.

\begin{tabular}{lrrcccc}
\hline \hline$f(\mathrm{~Hz})$ & 125 & 250 & 500 & 1000 & 2000 & 4000 \\
\hline$T_{20, \text { empty }}$ & 18.59 & 15.04 & 12.22 & 9.84 & 6.19 & 3.69 \\
$T_{20, \text { absorber }}$ & 6.79 & 3.64 & 3.29 & 3.25 & 2.83 & 2.08 \\
$\alpha_{T_{20}}$ & 0.37 & 0.75 & 0.8 & 0.74 & 0.70 & 0.75 \\
$\Delta \alpha$ & 0.49 & 0.49 & 0.34 & 0.29 & 0.28 & 0.23 \\
\hline \hline
\end{tabular}


TABLE IV. RTs $T_{20}$ in the undamped $\left(T_{20, \text { empty }}\right)$ and damped $\left(T_{20, \text { absorber }}\right)$ conditions, resulting absorption coefficient and deviation $\Delta \alpha .20$ panel diffusers are hung from the ceiling.

\begin{tabular}{lrrrrrr}
\hline \hline$f(\mathrm{~Hz})$ & 125 & 250 & 500 & 1000 & 2000 & 4000 \\
\hline$T_{20, \text { empty }}$ & 13.93 & 13.27 & 11.12 & 9.06 & 5.82 & 2.39 \\
$T_{20, \text { absorber }}$ & 5.59 & 2.77 & 2.39 & 2.37 & 2.08 & 1.62 \\
$\alpha_{T_{20}}$ & 0.39 & 1.03 & 1.18 & 1.12 & 1.11 & 1.16 \\
$\Delta \alpha$ & 0.47 & 0.21 & -0.04 & -0.09 & -0.13 & -0.18 \\
\hline \hline
\end{tabular}

diffusers. However, at high frequencies the absorption coefficient is overestimated compared to the theoretical values, although the deviation from $\alpha_{\text {theory }}$ is decreased compared to the case without diffusers in the chamber.

\section{Use of shortest decay time}

The proposed Bayesian framework is applied instead of the LR to estimate multiple decay parameters as described in Sec. III. Decay parameters are calculated for each octave band from $125 \mathrm{~Hz}$ to $4 \mathrm{kHz}$. In Fig. 4 the results for the decay times and intensities are plotted in the undamped (left) and damped (right) conditions without diffusing elements. In the entire frequency range two distinct peaks are detected, indicating a double-slope behaviour (i.e., a multi-exponential decay).

Table V shows the obtained absorption coefficients calculated in octave bands with Sabine's equation using the first peak from the estimated decay times. A good agreement between the theoretical and estimated absorption coefficients is achieved at the $250 \mathrm{~Hz}$ band and above. At the $125 \mathrm{~Hz}$ band the absorption coefficient obtained with the initial decay time is increased compared to the values obtained with $T_{20}$ from 0.37 to 0.48 . For better illustration, Fig. 5 shows the absorption coefficient as a function of frequency, calculated with $T_{20}$ with and without diffusers, and with the initial decay time $T_{1}$ without diffusers. The theoretical values are superimposed. Although no diffusers are present, the absorption coefficients obtained with the initial decay time estimated with the proposed method are in very good agreement with $\alpha_{\text {theory }}$ above the $250 \mathrm{~Hz}$ band. The absorption coefficient obtained with $T_{20}$ without diffusers is underestimated compared to the other values in the entire frequency range. With diffusers the absorption coefficient at the $500 \mathrm{~Hz}$ band and above is overestimated compared to the theoretical absorption coefficient.

In Table VI the relative error between the theoretical absorption coefficient and the calculated absorption coefficients with and without diffusing objects is summarized. The smallest deviation from $\alpha_{\text {theory }}$ is obtained with absorption coefficients calculated with the initial decay time with the proposed framework. The error between $\alpha_{T_{1}}$ and $\alpha_{\text {theory }}$ above the $250 \mathrm{~Hz}$ band is less than $5 \%$. At the $125 \mathrm{~Hz}$ band the theoretical absorption coefficient exceeds the calculated value. The deviation at $125 \mathrm{~Hz}$ could be due to the fact that the theoretical absorption coefficient is calculated by giving equal weights to all angles of incidence, whereas in the reverberation chamber it cannot be assumed that below the Schroeder frequency the incident sound on the absorber is uniformly distributed over all possible directions.
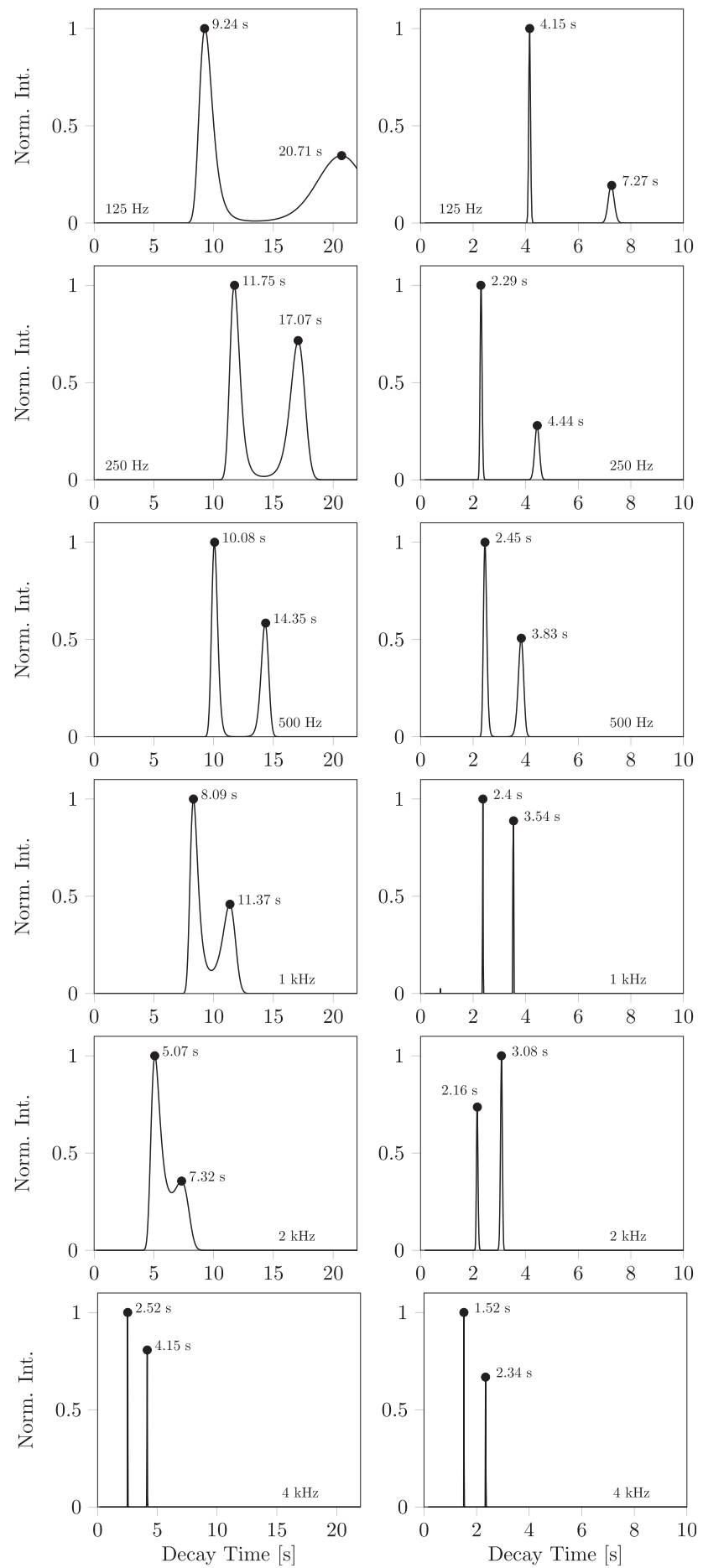

FIG. 4. Estimated normalized intensities of decay times from 125 to $4000 \mathrm{~Hz}$ for undamped (left) and damped (right) conditions and the corresponding peak values of the decay times. The first peak corresponds to the value used for calculating the absorption coefficient.

\section{DISCUSSION}

In this study, measurements are conducted in a boxshaped reverberation chamber with absorption concentrated on one of its walls, as such sound field allows separating the room modes into two groups ${ }^{3,16}$ (fast decaying and slow decaying), and thus provides a tractable environment to validate the methodology. Note however that additional mode groups (and therefore, additional decay times) can be considered to achieve higher accuracy, or in more complex environments. 
TABLE V. Initial decay times in the undamped $\left(T_{\text {empty }}\right)$ and damped ( $T_{\text {absorber }}$ ) conditions, resulting absorption coefficient $\alpha$, and deviation $\Delta \alpha$. No diffusers are installed.

\begin{tabular}{lrrrlll}
\hline \hline$f(\mathrm{~Hz})$ & 125 & 250 & 500 & 1000 & 2000 & 4000 \\
\hline$T_{\text {empty }}$ & 9.24 & 11.75 & 10.08 & 8.09 & 5.07 & 2.52 \\
$T_{\text {absorber }}$ & 4.15 & 2.29 & 2.45 & 2.4 & 2.16 & 1.52 \\
$\alpha$ & 0.48 & 1.26 & 1.11 & 1.05 & 0.96 & 0.94 \\
$\Delta \alpha$ & 0.38 & -0.02 & 0.03 & 0.02 & 0.05 & 0.04 \\
\hline \hline
\end{tabular}

It is widely accepted that without diffusing objects the sound field is not sufficiently diffuse to calculate the Sabine absorption coefficient. The results of this investigation indicate that reasonable absorption coefficients can be estimated without diffusing elements if the initial decay time, calculated via the Bayesian framework, is used. This means that the initial part of the decay process contains valuable information that should not be neglected. Care has to be taken at low frequencies, where the geometry of the room and the modal distribution has a great impact on the measured absorption coefficient. In practice, it cannot be assumed that the incident sound on the absorber is uniformly distributed over all possible directions. Therefore the measurement results in chambers of different volumes will differ from each other, depending on the modal distribution and the placement of the sample.

Predicting the influence of diffusing elements on the decay process is a very challenging task. Although it can be assumed that through the use of diffusers the grazing and non-grazing sound fields will be less distinct, the effect of adding diffusers seems to vary over the frequency range. When diffusing elements are introduced, the decay process can be regarded almost linear at mid and high frequencies, when an absorber is placed on the floor. However, the absorption coefficient is still underestimated in the low frequency range and overestimated in the mid and high frequency range compared to the theoretical absorption coefficient. In this investigation installing diffusers did not provide a solution to obtain reasonable absorption coefficients. As already

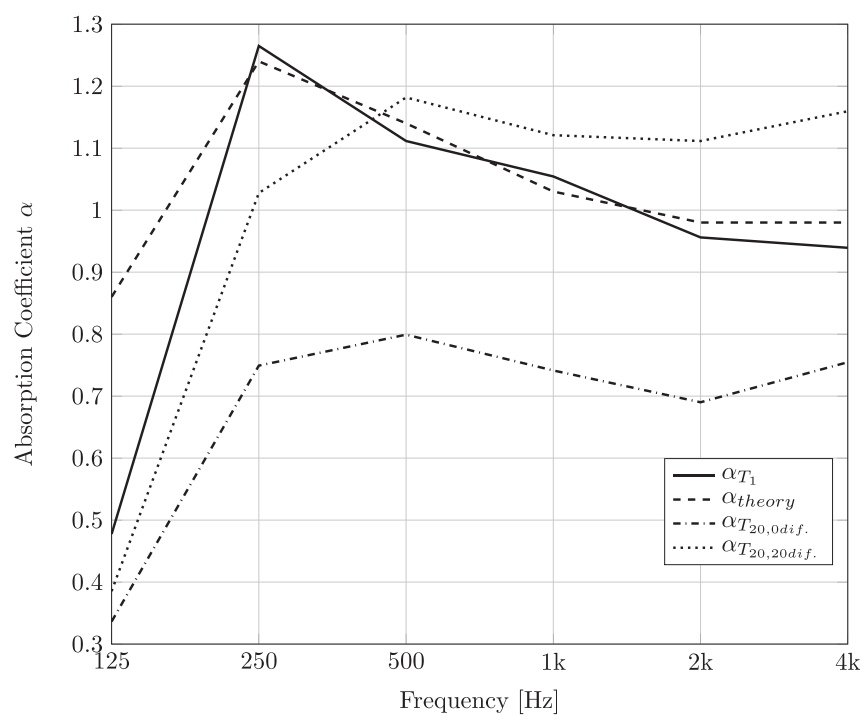

FIG. 5. Absorption coefficient $\alpha_{T_{1}}$ calculated with the shortest decay time compared to $\alpha_{\text {theory }}, \alpha_{T_{20,0 \text { dif. }}}$, and $\alpha_{T_{20,20 \text { dif. }}}$
TABLE VI. Relative error $\varepsilon$ in $\%$ between the theoretical absorption coefficient and the absorption coefficient calculated with reverberation parameter $T_{20}$ without (0 dif.) and with (20 dif.) diffusers, as well as with the decay time $T_{1}$ without ( 0 dif.) diffusers.

\begin{tabular}{lrrrrrr}
\hline \hline$f(\mathrm{~Hz}) / \varepsilon(\%)$ & 125 & 250 & 500 & 1000 & 2000 & 4000 \\
\hline$\varepsilon_{\alpha_{T_{20}, \text { odif. }}}$ & 56.98 & 39.52 & 29.82 & 28.16 & 28.57 & 23.47 \\
$\varepsilon_{\alpha_{T_{20}, 20 \text { dif. }}}$ & 54.65 & 16.94 & 3.51 & 8.73 & 13.27 & 18.37 \\
$\varepsilon_{\alpha_{T_{1}, \text { dif. }}}$ & 44.19 & 1.61 & 2.63 & 1.94 & 2.04 & 4.08 \\
\hline \hline
\end{tabular}

mentioned in the beginning, other researchers pointed out that diffusing elements introduce uncertainties that cannot be considered or modelled in an accurate way. ${ }^{3}$

It is important to point out that this work does not deal with the curvature of decays as a diffuseness measure, since the IRs used for the analysis were measured with an omnidirectional microphone which does not contain any directional information of the sound field. Recent work ${ }^{40}$ focuses on directional EDFs as a diffuseness measure. The quantification of the isotropy of the sound field is investigated in Ref. 41. Furthermore, this work does not deal with the quantification of double slopes like in Ref. 42, where ratios were calculated using linear fits with fixed ranges. Those methods are a rough estimation of whether or not a multi-exponential decay is present but they do not provide a solution to calculate accurate decay parameters.

\section{CONCLUSION}

This work has discussed the use of the initial decay time to calculate the absorption coefficient from measurements conducted in the reverberation chamber. Initial decay times as well as multiple decay parameters were estimated with a Bayesian framework from EDFs. The need for such a framework to estimate decay parameters from multiple-sloped decays as an alternative to LR has been argued with the fact that energy decays are not linear in most real world scenarios, especially in reverberation chambers at low frequencies and with uneven distribution of absorption.

Absorption coefficients calculated with the initial decay time were compared to the theoretical absorption coefficients calculated with a transfer matrix model combined with Miki's model including Thomasson's size correction. A good agreement was achieved in the frequency range from the $250 \mathrm{~Hz}$ band and above. In the $125 \mathrm{~Hz}$ band the theoretical absorption coefficient exceeded the calculated values due to the assumption of the sound incidence being uniformly distributed over all possible directions, which is not the case in the reverberation chamber. The results presented in this work included measurements without diffusing objects like panel or boundary diffusers, which is in contrast to the wellestablished approach in the reverberation chamber. The effect of diffusers on the decay process and on the sound field in general still poses a challenging task and such analysis is beyond the scope of this work.

Many round robin tests in the past have proven the poor interlaboratory reproducibility of absorption coefficient measurements. This work raises the question, if the poor 
reproducibility is to be owed to the evaluation of the decay parameters with LR. If a multi-exponential decay is present, using LR to obtain decay parameters will lead to questionable results. A future round robin test could evaluate if the method proposed in this paper provides a reliable alternative for measuring the Sabine absorption coefficient in reverberation chambers.

The proposed Bayesian method to obtain decay parameters is not restricted to laboratories. It is anticipated that the framework may also find important applications in measuring representative decay parameters in small and mid-sized room (e.g., in classrooms or offices, where absorption is concentrated on the ceiling).

\section{APPENDIX: BAYESIAN DECAY TIME ESTIMATION}

The mathematical formulations follow the derivations of Shukla et al. ${ }^{26-29}$ Brief parts of the acoustic application of the method have been published in Ref. 43, where different approaches for calculating decay parameters from multiexponential EDFs were compared.

The aim is to calculate the causal factors (decay terms with corresponding intensities) from a set of observations (EDFs) that produced them. The inverse problem can be written in the form of a Fredholm integral as

$$
D(y)=\int_{a}^{b} K(\tau, t) \Phi(\tau) d \tau+\nu(t)
$$

where $D(y)$ represents the measured data, $\Phi(\tau)$ is the intensity as a function of decay time (function to be obtained), and $\nu(t)$ denotes the noise in the data. The kernel function follows as:

$$
K(\tau, t)=\tau^{-1} e^{t / \tau}
$$

Equation (A1) can be written as a system of linear algebraic equations:

$$
d_{j}=\sum_{\mu=1}^{n_{\mathrm{mod}}} k_{j \mu} \phi_{\mu}+n_{j}, \quad j=1, \ldots, n_{\mathrm{dat}}
$$

and in matrix notation

$$
D=K \Phi+N
$$

where the indices dat and mod refer to the measured data and the model, respectively.

The proposed algorithm uses a quantified maximum entropy approach, estimating a positive additive distribution (PAD) from noisy and incomplete data. Knowing a number of possible solutions $A, B$, and $C$, one can describe them as conditional probabilities $\operatorname{pr}(A \mid D), \operatorname{pr}(B \mid D), \operatorname{pr}(C \mid D)$. If $\Phi$ was to represent a particular solution, one needs the probability distribution $\operatorname{pr}(\Phi \mid D)$ subject to $\Phi$. This is not directly obtainable from the given dataset $D$. However, the reversed conditioning $\operatorname{pr}(D \mid \Phi)$-better known as the likelihood function-is obtainable. The likelihood function can be written as

$$
\operatorname{pr}(D \mid \Phi)=\prod_{j=1}^{n_{\mathrm{dat}}} \frac{1}{\sqrt{2 \pi \sigma^{2}}} \exp \left(-\frac{1}{2 \sigma^{2}}\left[d_{j}-\sum_{\mu=1}^{n_{\mathrm{mod}}} k_{j \mu} \phi_{\mu}\right]^{2}\right)
$$

The model for estimating the unknown function can be found in Bayes theorem,

$$
\operatorname{pr}(\Phi \mid D)=\frac{\operatorname{pr}(\Phi) \operatorname{pr}(D \mid \Phi)}{\operatorname{pr}(D)}
$$

with $\operatorname{pr}(D)$ being a normalizing factor to ensure that the sum of the probabilities of all possible solutions is equal to one. This formula represents one of the most relevant mathematical fundamentals of data analysis. Considering the $\operatorname{pr}(D \mid \Phi)$ as given and $\operatorname{pr}(D)$ being a normalizing constant, the only remaining unknown term is the prior probability distribution of $\Phi$, denoted as $\operatorname{pr}(\Phi)$. The pointwise probability

$$
\operatorname{pr}(\Phi \mid m, \alpha) \propto \exp ((\alpha S(\Phi, m))
$$

reflects the most important part of the quantified entropic prior. ${ }^{44}$ The two parameters $m$ and $\alpha$ represent a model for $\Phi$ and an inverse measure of the expected spread of values of $\Phi$ about $m$. The pointwise joint probability distribution is given by

$$
\begin{aligned}
\operatorname{pr}(\Phi, D \mid m, \alpha)= & \left(\frac{\alpha}{2 \pi}\right)^{r / 2} \prod \frac{1}{\sqrt{2 \pi \sigma^{2}}} \\
& \times \exp \left(\alpha S(\Phi, m)-\frac{1}{2} C(\Phi, D)\right),
\end{aligned}
$$

where the function $S(\Phi, m)$ denotes the Shannon-Jaynes entropy. The pointwise joint probability is proportional to the posterior probability by

$$
\operatorname{pr}(\Phi \mid m, \alpha, D)=\frac{\operatorname{pr}(\Phi, D \mid m, \alpha)}{\operatorname{pr}(D \mid m, \alpha)}
$$

Since the Shannon-Jaynes entropy is a convex function with negative definite curvature and $C(f)$ a positive curvature, the posterior probability $\operatorname{pr}(\Phi \mid m, \alpha, D)$ has a unique maximum at

$$
\alpha \frac{\partial S}{\partial \Phi}-\frac{1}{2} \frac{\partial C}{\partial \Phi}=0 \quad \text { at } \quad \Phi=\hat{\Phi} .
$$

The obtained distribution $\hat{\Phi}$ is the single most probable PAD. The choice of a well-guessed kick-off solution is essential as it has a stabilizing and regularizing effect. Furthermore it shortens the time needed for the solution to converge. For this particular reason the algorithm uses a general optimal linear filter to compute a good kick-off solution. Designing a filter in this case means constructing a filter matrix $F$ to solve the inverse problem by satisfying a minimization criterion. This is chosen to be the mean squared error between the "real" $\Phi$ and the one extracted by the filter $F$, 


$$
\sum_{v} p_{v}\left\langle\left|F\left(K \Phi_{v}-D\right)\right|^{2}\right\rangle_{N}=\min
$$

To obtain the coefficients of the filter $F$, the left side of Eq. (A11) has to be differentiated with respect to $F$ and then equated to zero to satisfy the minimization criterion. This leads to a filter $F$ to obtain the regularized solution

$$
\Phi_{r}=F D,
$$

with

$$
F=\frac{C_{\Phi} K^{T}}{K C_{\Phi} K^{T}+C_{N}},
$$

where

$$
C_{\Phi}=\sum_{v} p_{v} \Phi_{v} \Phi_{v}^{T} ; \quad C_{N}=\left\langle N N^{T}\right\rangle .
$$

${ }^{1}$ ISO 354:2003, "Acoustics-Measurement of sound absorption in a reverberation room" (International Organization for Standardization, Geneva, Switzerland, 2003).

${ }^{2}$ W. Sabine, Collected Papers on Acoustics (Harvard University Press, Cambridge, 1922), pp. 3-68.

${ }^{3}$ F. V. Hunt, L. L. Beranek, and D. Y. Maa, "Analysis of sound decay in rectangular rooms,” J. Acoust. Soc. Am. 11(1), 80-94 (1939).

${ }^{4} \mathrm{H}$. Kuttruff, "Eigenschaften und Auswertung von Nachhallkurven (Characteristics and analysis of decay curves)," Acta Acust. Acust. 8(4), 273-280 (1958).

${ }^{5}$ E. Nilsson, "Decay processes in rooms with non-diffuse sound fields. Part I: Ceiling treatment with absorbing material," Build. Acoust. 11(1), 39-60 (2004).

${ }^{6} \mathrm{~W}$. Davern and P. Dubout, "First report on Australasian comparison measurements of sound absorption coefficients," DBR Special Report, CSIRO Division of Building Research, Highett (1980).

${ }^{7}$ ASTM, "Interlaboratory study to establish precision statements for ASTM C423," Research Report E33-1010 (2006).

${ }^{8}$ A.-C. Thysell, "Test codes for suspended ceilings-Sound absorption RRT," Tyrens AB project no. 224628, Tyrens, A. B. Sweden (2011).

${ }^{9}$ L. Savioja and U. P. Svensson, "Overview of geometrical room acoustic modeling techniques," J. Acoust. Soc. Am. 138(2), 708-730 (2015).

${ }^{10} \mathrm{M}$. Vorländer, "Computer simulations in room acoustics: Concepts and uncertainties," J. Acoust. Soc. Am. 133(3), 1203-1213 (2013).

${ }^{11}$ J. Allard, Propagation of Sound in Porous Media: Modelling Sound Absorbing Materials (Elsevier Science Publishers, Essex, England, 1993), p. 38.

${ }^{12}$ Y. Miki, “Acoustical properties of porous materials. Modifications of Delany-Bazley models," J. Acoust. Soc. Am. 11(1), 19-24 (1990).

${ }^{13}$ S.-I. Thomasson, "On the absorption coefficient," Acta Acust. Acust. 44(4), 265-273 (1980).

${ }^{14}$ M. R. Schroeder, "New method of measuring reverberation time," J. Acoust. Soc. Am. 37(3), 409-412 (1965).

${ }^{15} \mathrm{P}$. Bruel, "The enigma of sound power measurements at low frequencies," Bruel \& Kjaer Tech. Rev. 34, 3-40 (1978).

${ }^{16}$ F. Jacobsen, Fundamentals of General Linear Acoustics (John Wiley \& Sons, United Kingdom, 2013), pp. 151-153.

${ }^{17} \mathrm{~F}$. Jacobsen, "Decay rates and wall absorption at low frequencies," J. Sound Vib. 81(3), 405-412 (1982).

${ }^{18}$ ISO 3382-2:2008, "Acoustics-Measurement of room acoustic parameters-Part 2: Reverberation time in ordinary rooms" (International Organization for Standardization, Geneva, Switzerland, 2008).

${ }^{19}$ E. Nilsson, "Decay processes in rooms with non-diffuse sound fields. Part II: Effect of irregularities,” Build. Acoust. 11(2), 133-143 (2004).
${ }^{20}$ J. Balint, F. Muralter, M. Nolan, and C.-H. Jeong, "Energy decay curves in reverberation chambers and the influence of scattering objects on the absorption coefficient of a sample," in Conference Proceedings Euronoise 2018, Crete, Greece (2018), pp. 2025-2030.

${ }^{21}$ J. Balint and G. Graber, "Gekruemmte Abklingkurven in Hallräumen (Curved energy decays in reverberation rooms)," in Proceedings of the 44th Annual Meeting for Acoustics DAGA, Munich, Germany (2018).

${ }^{22}$ N. Xiang and P. M. Goggans, "Evaluation of decay times in coupled spaces: Bayesian parameter estimation," J. Acoust. Soc. Am. 110(3), 1415-1424 (2001).

${ }^{23}$ N. Xiang and P. M. Goggans, "Evaluation of decay times in coupled spaces: Bayesian decay model selection," J. Acoust. Soc. Am. 113(5), 2685-2697 (2003).

${ }^{24} \mathrm{~N}$. Xiang and T. Jasa, "Evaluation of decay times in coupled spaces: An efficient search algorithm within the Bayesian framework," J. Acoust. Soc. Am. 120(6), 3744-3749 (2006).

${ }^{25}$ N. Xiang, P. Goggans, T. Jasa, and P. Robinson, "Bayesian characterization of multiple-slope sound energy decays in coupled-volume systems," J. Acoust. Soc. Am. 129(2), 741-752 (2011).

${ }^{26}$ A. Shukla, M. Peter, and L. Hoffmann, "Analysis of positron lifetime spectra using quantified maximum entropy and a general linear filter," Nuclear Instrum. Methods Phys. Res. A 335, 310-317 (1993).

${ }^{27}$ A. Shukla, L. Hoffmann, A. Manuel, and M. Peter, "Bayesian methods for lifetime analysis," Mater. Sci. Forum 175-178, 939-946 (1995).

${ }^{28}$ A. Shukla, L. Hoffmann, A. Manuel, and M. Peter, "Melt 4.0a a Program for Positron Lifetime Analysis," Mater. Sci. Forum 255-257, 233-237 (1997).

${ }^{29}$ L. Hoffmann, A. Shukla, M. Peter, B. Barbiellini, and A. Manuel, "Linear and non-linear approaches to solve the inverse problem: Applications to positron annihilation experiments," Nuclear Instrum. Methods Phys. Res. Sec. A 335(1-2), 276-287 (1993).

${ }^{30}$ C.-H. Jeong, S.-H. Choi, and I. Lee, "Bayesian inference of the flow resistivity of a sound absorber and the room's influence on the Sabine absorption coefficients," J. Acoust. Soc. Am. 141(3), 1711-1714 (2017).

${ }^{31} \mathrm{~N}$. Xiang and C. Landschoot "Bayesian inference for acoustic direction of arrival analysis using spherical harmonics," Entropy 21(579), 2-21 (2019).

${ }^{32}$ S. F. Gull and J. Skilling, "Maximum entropy method in image processing," IEE Proc. F 131(6), 646-659 (1984).

${ }^{33}$ R. K. Bryan, Maximum Entropy and Bayesian Methods, edited by P. F. Fougere (Kluwer, Dordrecht, 1990), Vol. 127(6), pp. 221-232.

${ }^{34}$ H. Kuttruff, Room Acoustics (CRC Press, Boca Raton, FL, 2016), p. 302.

${ }^{35}$ C.-H. Jeong, "Non-uniform sound intensity distributions when measuring absorption coefficients in reverberation chambers using a phased beam tracing," J. Acoust. Soc. Am. 127(6), 3560-3568 (2010).

${ }^{36}$ C.-H. Jeong, "Converting Sabine absorption coefficients to random incidence absorption coefficients," J. Acoust. Soc. Am. 133(6), 3951-3962 (2013).

${ }^{37}$ J. Brunskog, "The forced sound transmission of finite single leaf walls using a variational technique," J. Acoust. Soc. Am. 132(3), 1482-1493 (2012).

${ }^{38}$ J. Brunskog, "Sound radiation from finite surfaces," J. Acoust. Soc. Am. 133(5), 3385-3385 (2013).

${ }^{39}$ M. E. Delany and E. N. Bazley, "Acoustical properties of fibrous absorbent materials," Appl. Acoust. 3(2), 105-116 (1970).

${ }^{40} \mathrm{M}$. Berzborn and M. Vorländer, "Investigations on the directional energy decay curves in reverberation rooms," in Conference Proceedings Euronoise 2018, Crete, Greece (2018), pp. 2005-2010.

${ }^{41}$ M. Nolan, E. Fernandez-Grande, J. Brunskog, and C.-H. Jeong, “A wavenumber approach to quantifying the isotropy of the sound field in reverberant spaces," J. Acoust. Soc. Am. 143(4), 2514-2526 (2018).

${ }^{42}$ D. T. Bradley and L. M. Wang, "Quantifying the double slope effect in coupled volume room systems," Build. Acoust. 16(2), 105-123 (2009).

${ }^{43} \mathrm{~F}$. Muralter and J. Balint, "Analysis tools for multi-exponential energy decay curves in room acoustics," in Proceedings of the 45th Annual Meeting for Acoustics DAGA, Rostock, Germany (2019).

${ }^{44}$ J. Skilling, "Quantified maximum entropy," in Maximum Entropy and Bayesian Methods (Springer, New York, 1990), pp. 341-350. 